\title{
Predicting patterns of failure in temporal lobe GBMs: possible implications on radiotherapy treatment portals
}

\author{
Dasantha Jayamanne ${ }^{1,4^{*}}$ (D), Helen Wheeler ${ }^{1,2,5}$, David Brazier ${ }^{3}$, Allison Newey $^{3}$, Marina Kastelan ${ }^{1,5}$, Linxin Guo $^{1}$
} and Michael Back ${ }^{1,2,4,5}$

\begin{abstract}
Background: Characterise patterns of failure of Temporal Lobe (TL) Glioblastoma (GBM) following treatment with relation to normal temporal lobe anatomy and neural pathways.

Methods: 335 GBM patients received radiotherapy between 03/2007 and 07/2014, 100 were located in TL. Site of initial tumour and subsequent relapse were subdivided into 5 local TL sites (anterior, lateral, medial, posterior and superior); 5 adjacent regional sites (occipital lobe, inferior frontal lobe, caudate/thalamus/internal/external capsules, fornix/ventricular trigone), and 5 distant failure sites (ventricles, contralateral hemisphere, brainstem, leptomeninges and spine). Extension along major neuroanatomical pathways at initial presentation and at first documented Magnetic Resonance Imaging (MRI) failure were categorised into anterior, superior, medial and posterior pathways.

Results: Of the 100 patients, 86 had radiological progress with a median survival of 17.3 months. At initial diagnosis, $74 \%$ of tumours were linked to one TL site and $94 \%$ were confined to the TL. 19\% had neural pathway disease at initial pre-treatment MRI. At first recurrence locoregional site failure was $74 \%$. 26\% failed within distant sites and 53\% patients were noted to have neural pathway involvement.

Initial tumour location predicted for local site recurrence $(p<0.0001)$, regional site recurrence $(p=0.004)$ and neural pathway recurrence pattern ( $p=0.005)$, but not for distant sites $(p=0.081$ ).

Conclusion: Most GBMs fail at local or adjacent regional sites. Many of the recurrences occurred in a predictable pattern within a local or regional site, unique to initial TL site with more than half involving neural pathways.

Knowledge of tumour infiltration and failure may improve target definition and radiotherapy.
\end{abstract}

Keywords: Glioblastoma, Radiation, Recurrence, Patterns of failure

\section{Background}

Glioblastoma (GBM) is the most common primary malignant brain tumour and despite subtle advances in the past decade, survival remains poor beyond three years. Current standard of practice involves aggressive surgical resection followed by concurrent chemoradiotherapy followed by adjuvant chemotherapy [1]. Despite aggressive treatment, the prognosis for most patients is short and with a median survival of 14-18 months [2-4]. Several other therapeutic

\footnotetext{
* Correspondence: Dasantha.Jayamanne@health.nsw.gov.au

${ }^{1}$ Northern Sydney Cancer Centre, Royal North Shore Hospital, Sydney, NSW, Australia

${ }^{4}$ Central Coast Cancer Centre, Gosford Hospital, Gosford, NSW, Australia Full list of author information is available at the end of the article
}

agents have been trialed in the adjuvant setting with no clear survival benefit $[5,6]$. As such, there is a need to improve current treatment protocols. Glioblastoma tumor spread along white matter tract as found in the temporal lobe, could be one explanation of the high loco-regional recurrence rate of glioblastoma and thereby poor overall survival after standard therapy.

Radiotherapy has been shown to improve survival in malignant gliomas [7]. However, despite aggressive local treatment, with surgery and radiotherapy, $80 \%$ of gliomas fail within $2 \mathrm{~cm}$ of the resection cavity [8-11], Older studies evaluating hyperfractionated dose escalation show no improvement in tumour control or survival $[12,13]$. Others have shown better control with accelerated very high doses

(c) The Author(s). 2018 Open Access This article is distributed under the terms of the Creative Commons Attribution 4.0 International License (http://creativecommons.org/licenses/by/4.0/), which permits unrestricted use, distribution, and 
of radiotherapy, up to 90Gy, but with unacceptable radiation necrosis [14]. Clearly, a greater understanding of tumour biology, patterns of failure and infiltration is needed to improve tumour control.

Glioma infiltration along white matter tracts has been proposed as a potential means of spread of tumour both locally and distantly within the brain [15]. Standard imaging techniques with computed tomography and magnetic resonance imaging (MRI) do not adequately delineate these regions. T2-weighted MRI Fluid Attenuated Inversion Recovery FLAIR sequence have been shown to characterise early glioblastoma infiltration and have been incorporated into post-operative planning of radiation therapy (4) and assessment of Glioma progression by Response Assessment in Neuro-oncology (RANO) criteria $[16,17]$. However, precise anatomical localisation of tumour within these sequences is yet to be established. Newer functional imaging with positron emission tomography (PET) tracers, especially $18 \mathrm{~F}$-fluoroethyl-L-tyrosine (FET) PET [18-20], have shown some improvements in delineation of glioma but a highly sensitive and specific tracer is yet to be established [21].

Advanced MRI based tractography using diffusion tensor imaging (DTI) is sensitive in detecting the direction of water diffusion along white matter tracts. Studies have shown peri-tumoural changes in DTI that were not apparent in conventional MRI [22] and others have shown biopsies within abnormal white matter tracts seen on DTI have yielded active tumour [23]. Further to this, failure along white matter tracts has been associated with inferior outcomes [24], presumably due to a more infiltrative tumour biology.

Due to the uncertainties of tumour infiltration, most radiotherapy protocols use generous uniform or isotropic margins to account for microscopic tumour infiltration $[25,26]$. However, this may lead to high doses of radiation to normal uninvolved brain tissue and contribute to long-term neurological toxicity such as cognitive, memory and functional abnormalities.

This study aims to characterise the failure pattern of treated temporal lobe (TL) glioblastoma on standard sequence MRI by comparing initial tumour location to the site of first failure, including potential infiltration along neural pathways.

\section{Methods}

\section{Patient population}

Adult patients with a new diagnosis of primary brain tumour referred to the Department of Radiation Oncology at the Northern Sydney Cancer Centre after May 2007 were entered into a prospective database, approved by the local Institutional Ethics Review Board. All patients with GBM involving the TL managed with Radiotherapy (RT) were retrospectively analysed.
Patients aged $<75$ with good performance status received treatment consistent with the European Organisation for Research and Treatment of Cancer- National Cancer Institute of Canada (EORTC-NCIC) [1] Protocol, whilst elderly patients (aged $>75$ years) or those with impaired Eastern Cooperative Oncology Group (ECOG) status were managed with hypofractionated RT (40Gy in 15 fractions) with or without systemic therapy. All patients were treated with Intensity Modulated Radiotherapy (IMRT) by one subspecialised radiation oncologist.

Patient, tumour and treatment factors were recorded into a prospective database. Performance status was assessed at the start of RT with the ECOG scale.

\section{Follow-up}

All patients were followed closely with initial MRI at one month post RT $(M+1)$ then second monthly MRI until completion of adjuvant temozolomide or for six months, if not receiving chemotherapy, then three monthly until end of year 3 post RT, then four-six monthly until progression. Standard MRI sequences where obtained including T1/T2 weighted images pre and post contrast. T2 FLAIR sequences, Diffusion Weighted Imaging (DWI) and Apparent Diffusion Coefficient (ADC) sequences were also routinely acquired.

\section{Initial tumour location}

Site of initial tumour location at time of diagnosis was recorded and categorized into five TL sites; anterior, lateral, medial, posterior and superior (Fig. 1). Tumours involving regional sites past the temporal lobe were also categorized into 5 adjacent sites including the occipital lobe, inferior frontal lobe, caudate/internal/external capsules, fornix and thalamus/trigone of lateral ventricle (Fig. 2). Distant tumour involvement, where present, was classified into ventricular, contralateral hemisphere, brainstem, leptomeninges and spine. Tumours overlapping and involving multiple sites were coded for each site of involvement and analysed accordingly.

\section{Neural pathway involvement}

Using established neural pathways atlases [27], potential neural pathway involvement was noted. Potential tumour involvement was classified by clinician discretion if within close approximation or seemingly tracking along neural pathways (Fig. 3). Specifically the pathways were superior (uncinate fasciculus), anterior (inferior longitudinal fasciculus), medial (fornix, cingulum) and posterior (inferior fronto-occipital fasciculus).

\section{Recurrent tumour}

Recurrence was noted as the date of first MRI scan showing recurrent tumour or clinical deterioration not thought to be pseudoprogression. Recurrence was 


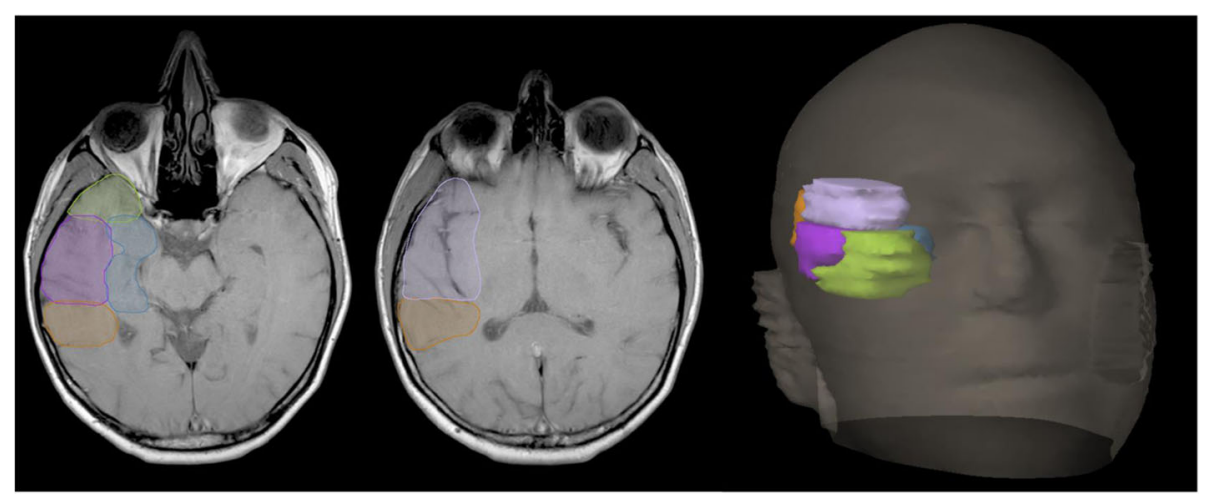

Fig. 1 Local temporal lobe sites: anterior (green), lateral (purple), medial (blue), posterior (orange) and superior (lilac)

defined based on careful review of subsequent MRI from date of suspected MRI recurrence and any worsening clinical symptoms in line with RANO Working Group criteria [17]. All patients were discussed in a multidisciplinary tumour board meeting (MDT) with the involvement of treating physicians, diagnostic radiologists and nuclear medicine physicians. At time of recurrence, tumour location was noted and classified into local, regional, distant and pathway failure as per the above classifications.

\section{Statistical evaluation}

Chi-Square test of independence was conducted on all patients to test if an association existed between the initial tumour location and the sites of first recurrence or neural pathway involvement. Pretreatment prognostic factors including age, extent of resection, Recursive partitioning analysis (RPA) Class and performance status were analysed with Kaplan-Meier survival analysis and log rank test to calculate the median survival for each sub-group and $p$-value to determine if any significant difference could be found. Cox regression model was used to analyse the impact of pretreatment prognostic factors on time to tumour recurrence. All reported $p$ values were two-tailed. Statistical significance was defined as $p \leq 0.05$. IBM SPSS Statistics Version 24 was used for statistical analysis.

\section{Results}

335 consecutive GBM patients received IMRT between March 2007 and July 2014. 100 patients had tumours located within the TL. Of these, 86 had radiological progression and were included in the study. The median progression free survival was 7.1 months (95\% CI 5.38.7) with an overall survival of 17.3 months (95\% CI 13.8-18.1). The individual patient characteristics have been summarised in Table 1.

\section{Initial tumour location}

At initial diagnosis 81/86 (94\%) patients had tumours confined to the TL only, with $3(4 \%)$ patients having regional involvement (occipital lobe, fornix and trigone of lateral ventricle) and $2(2 \%)$ patients having distant disease (ventricles). Of the patients with TL disease only, 60/81 (74\%) had tumours localised to within one TL subsite. 16 (19\%) had neural pathway disease at initial pre-treatment MRI.

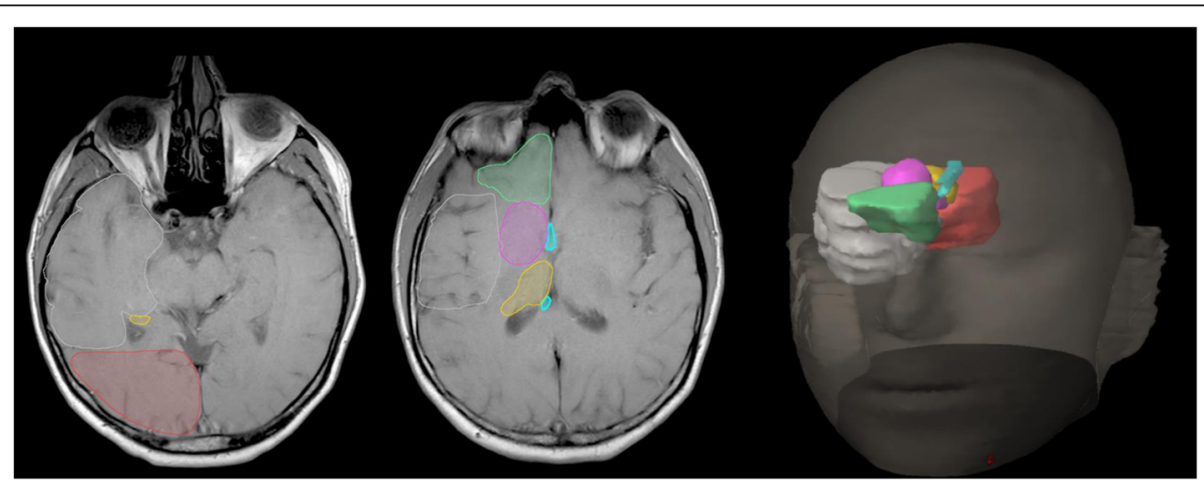

Fig. 2 Five regional sites adjacent to temporal lobe (white): occipital lobe (red), inferior frontal lobe (green), caudate/internal/external capsules (pink), fornix (cyan) and thalamus/trigone of lateral ventricle (orange). Temporal lobe (white) 


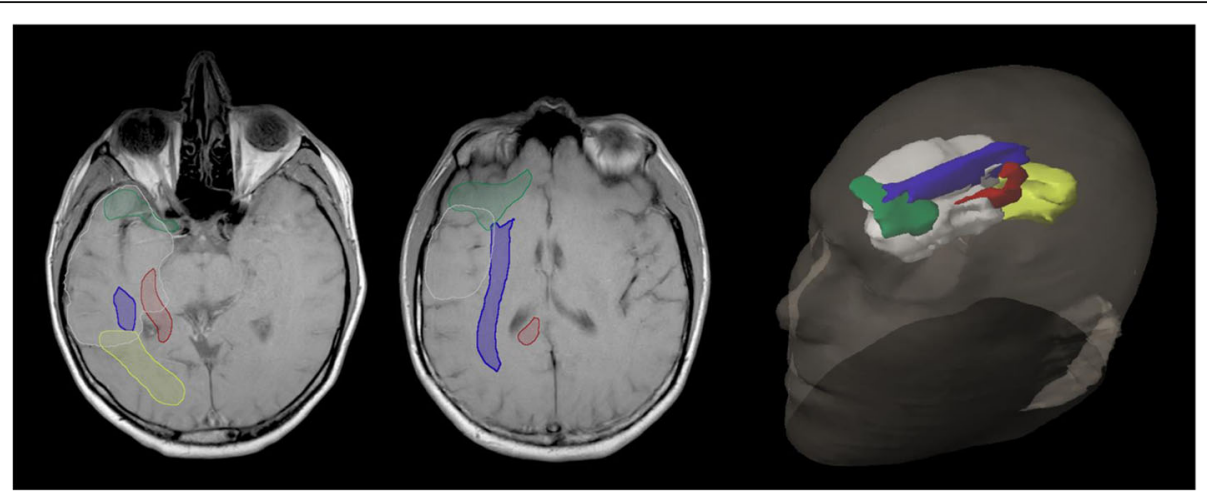

Fig. 3 Four neural pathways adjacent to the temporal lobe (white): superior (green), anterior (blue), medial (red) and posterior (yellow)

\section{Tumour recurrence site}

The majority of relapses involved the TL with 72 patients (84\%) having a component of local failure, of which 22 (26\%) had involvement of multiple TL sites. Involvement of regional sites (48\%) and distant sites (26\%) also occurred at initial relapse. 46 patients (53\%) patients were noted to have neural pathway involvement at time of recurrence (Table 2). In regards to isolated relapses, 35 patients $(41 \%)$ had relapse confined to the TL alone; 64 (74\%) both local and regional sites; 3 (3\%) in regional sites; $14(16 \%)$ regional and distant sites and $8(9 \%)$ were distant relapses only.

Table 1 Patient characteristics

\begin{tabular}{ll}
\hline Characteristics & Patients (Percentages) \\
\hline Gender: & $49(57)$ \\
Male & $37(43)$ \\
Female & 63 \\
Median Age: & \\
Surgery: & $7(8)$ \\
Biopsy & $32(37)$ \\
STR & $47(55)$ \\
GTR & \\
Adjuvant treatment: & $65(75)$ \\
Long course adjuvant RT/TMZ & $4(5)$ \\
Hypofractionated RT/TMZ & $17(20)$ \\
Hypofractionated RT & $0(0)$ \\
TMZ alone & \\
Radiotherapy dose: & $65(76)$ \\
60Gy & $21(24)$ \\
$40 G y$ & $70(81)$ \\
Re-resection rates: & $16(19)$ \\
No further craniotomy & \\
Re-resection & \\
\hline
\end{tabular}

GTR - Gross Tumour Resection

STR - Sub-Total Resection

TMZ - Temazolamide

\section{Predictable sites of recurrence}

Initial TL site location predicted for local site recurrence $(p<0.0001)$, regional site recurrence $(p=0.004)$ and neural pathway recurrence pattern $(p=0.005)$, but not for distant sites $(p=0.081)$. Figure 4 shows distribution of local and regional recurrences dependent on initial origin of tumour within the TL and Fig. 5 shows neural pathway recurrences.

\section{Prognostic factors for relapse pattern}

For the whole group of TL tumours, median survival was improved for patients managed with higher dose RT of 60Gy versus 40Gy with 17 months and 11 months respectively $(p=0.01, \mathrm{HR}=0.489,95 \% \mathrm{CI}=[0.274,0.871])$ but not with age $(p=0.17, \mathrm{HR}=1.486,95 \% \mathrm{CI}=[0.835$, $2.646])$, resection type $(p=0.24, \mathrm{HR}=0.751,95 \% \mathrm{CI}$ $=[0.464,1.218])$, ECOG Performance Status $(p=0.11, \mathrm{HR}$ $=1.617,95 \% \mathrm{CI}=[0.889,2.942])$, and RPA Class $(p=0.1$, $\mathrm{HR}=2.14,95 \% \mathrm{CI}=[0.851,5.38])$.

Assessment of local, regional and distant pattern of failure was undertaken with respect to prognostic factors mentioned above using cox regression modeling. On Univariate analysis, patients with sub-total resection (STR) or biopsy had a higher rate of regional failure $(p=0.002, \mathrm{HR}=2.966,95 \% \mathrm{CI}$ $=[1.468,5.992])$, but no other significant difference were seen between prognostic factors and local or distant failures. No statistical differences were seen on multivariate analysis.

The influence of extent of resection and failure site is described further in Table 3. Specifically, extent of resection did not predict for local failure with $86 \%$ of patients with gross total resection (GTR) failing at initial local site compared to $80 \%$ with subtotal resection or biopsy $(p=0.40)$. However those patients with subtotal resection or biopsy had a greater proportion of regional (63\% vs 33\%: $p=0.01$ ) and distant failure ( $43 \%$ vs $18 \%: p=0.01)$ than those with GTR. When regional failure occurred, it developed earlier in patients with subtotal resection than those with GTR 
Table 2 Sites of recurrence of temporal lobe tumours

\begin{tabular}{|c|c|c|c|c|c|c|c|}
\hline \multirow{2}{*}{ Anterior/Pole: $n=22$} & \multicolumn{2}{|c|}{ Local recurrence: } & \multirow{2}{*}{\multicolumn{2}{|c|}{$\begin{array}{l}\text { Reg recurrence: } \\
\text { Inferior Frontal Lobe }\end{array}$}} & \multirow{2}{*}{$\begin{array}{l}\text { Distant recurrence: } \\
\text { Ventricle }\end{array}$} & \multicolumn{2}{|c|}{ Neural pathway recurrence: } \\
\hline & Anterior & Superior & & & & Superior & \\
\hline & $11(50 \%)$ & $10(45 \%)$ & $6(27 \%)$ & & $3(13 \%)$ & $11(50 \%)$ & \\
\hline \multirow[t]{2}{*}{ Lateral: $n=32$} & \multicolumn{2}{|l|}{ Lateral } & \multicolumn{2}{|c|}{ Tail of Caudate } & Contralateral Hemisphere & Superior & \\
\hline & \multicolumn{2}{|l|}{$28(87.5 \%)$} & \multicolumn{2}{|l|}{$11(34 \%)$} & $2(6 \%)$ & $6(19 \%)$ & \\
\hline \multirow[t]{2}{*}{ Medial/Hippocampal: $n=15$} & \multicolumn{2}{|l|}{ Medial } & Fornix & Occipital & Ventricle & Medial & Posterior \\
\hline & \multicolumn{2}{|l|}{$15(65 \%)$} & $7(30 \%)$ & $6(26 \%)$ & $9(39 \%)$ & $10(43 \%)$ & $7(30 \%)$ \\
\hline \multirow[t]{2}{*}{ Posterior: $n=15$} & \multicolumn{2}{|l|}{ Posterior } & \multicolumn{2}{|l|}{ Occipital } & Ventricle & Posterior & \\
\hline & \multicolumn{2}{|l|}{$12(80 \%)$} & \multicolumn{2}{|l|}{$8(53 \%)$} & $4(27 \%)$ & $8(53 \%)$ & \\
\hline \multirow[t]{2}{*}{ Superior/Insular: $n=17$} & \multicolumn{2}{|l|}{ Superior } & \multicolumn{2}{|c|}{ Inferior Frontal } & & Superior & \\
\hline & \multicolumn{2}{|l|}{15 (88\%) } & \multicolumn{2}{|l|}{$4(24 \%)$} & $0(0 \%)$ & $8(47 \%)$ & \\
\hline
\end{tabular}

$(\mathrm{p}=0.01)$. Isolated regional or distant failure occurred in 14 patients and this was not influenced by extent of resection $(p=0.44)$.

There was no significant difference between site of relapse and time of death from relapse. The median for the total group was 6 months, and there was no difference between failures at local, regional or distant sites $(p=0.65)$.

\section{Outcome for neural tract relapse}

Initial neural pathway involvement was present in 16 patients (19\%) and was associated with a subsequent greater rate of subtotal resection. At time of relapse, 46 patients (53\%) were noted to have neural pathway involvement. For patients with subtotal resection there was a trend for subsequent earlier tract failure with a median time of 10 months compared to 19 months, which did not reach statistical significance $(p=0.06)$. There was no one neural pathway that was associated with the 14 patients with distant only failure $(p=0.34)$.

In regards time from neural tract involvement at failure and death, the median time to death was 7 months compared to 4 months in those without neural tract failure, and this did not reach statistical significance $(p=0.35)$.

\section{Discussion}

Our results show that despite good initial tumour resection, with GTR and STR achieved in 55 and 37\% respectively, $74 \%$ of patients had first documented failure isolated to local and adjacent brain sites. Although our study involved temporal lobe tumours only, others have shown similar rates of local failure elsewhere in the brain [10$12]$, suggesting that this is not unique to this anatomical location alone. Historically, this has been the case in the pre-temozolomide era, with dose escalations studies of up to 90 Gy showing high infield failure rates of $89-91 \%$ [9, 28]. More recently, the use of temozolomide has become standard of practice since EORTC-NCIC protocol, especially in good performance status patients under the age of 70, showing an improvement in median survival of 14.6 months and an improvement in 5 year survival with concurrent and adjuvant temozolomide of $9.8 \%$ compared with $1.9 \%$ radiotherapy alone [29].

Despite these improvements in overall survival, modern series still show a failure rate of $70-85 \%$ local failure rate within $2-3 \mathrm{~cm}$ of the tumour cavity or directly within the high dose radiotherapy portals $[9,10,28,30,31]$. Some studies have reported a change in failure pattern in patients with GBM and 06-methylguanine DNA methyltransferase (MGMT) methylated tumours. Brandes et al. [31] reviewed 95 patients treated with high dose radiotherapy who showed an infield or marginal recurrence rate of $85 \%$ in unmethylated MGMT tumours, compared with $57.9 \%$ of patients with methylated tumours; with failures out of field presenting at significantly longer time intervals to those with in field failures. Therefore, knowledge of anatomical regions of failure and possible mechanisms of tumour spread may aid in improving tumour control in these patients.

There is uncertainty as to the optimal margins for adjuvant radiotherapy in GBM. The EORTC uses a single-phase technique in which the gross tumour volume (GTV) is defined as the surgical tumor bed plus any residual enhancing tumor. This is expanded by $2 \mathrm{~cm}$ to create the clinical target volume (CTV) and another $0.3-0.5 \mathrm{~cm}$ for the planning target volume (PTV) [32]. The Radiation Therapy Oncology Group (RTOG) protocols use a two phase technique with the first phase encompassing the primary contrast enhancing tumour and peri-tumoural oedema with a $2-2.5 \mathrm{~cm}$ expansion treated to a dose of $46 \mathrm{~Gy}$. The second phase is purely the contrast enhancing tumour with an expansion of $2-2.5 \mathrm{~cm}$ to an additional dose of 14Gy. However, the use of isotropic marginal expansions such as these may lead to overtreatment of normal brain tissue with no clear advantage in local control or survival. High dose radiotherapy to brain tissue has been shown to increase neurotoxic side effects including radionecrosis and cognitive decline [33, 34]. The volume of 


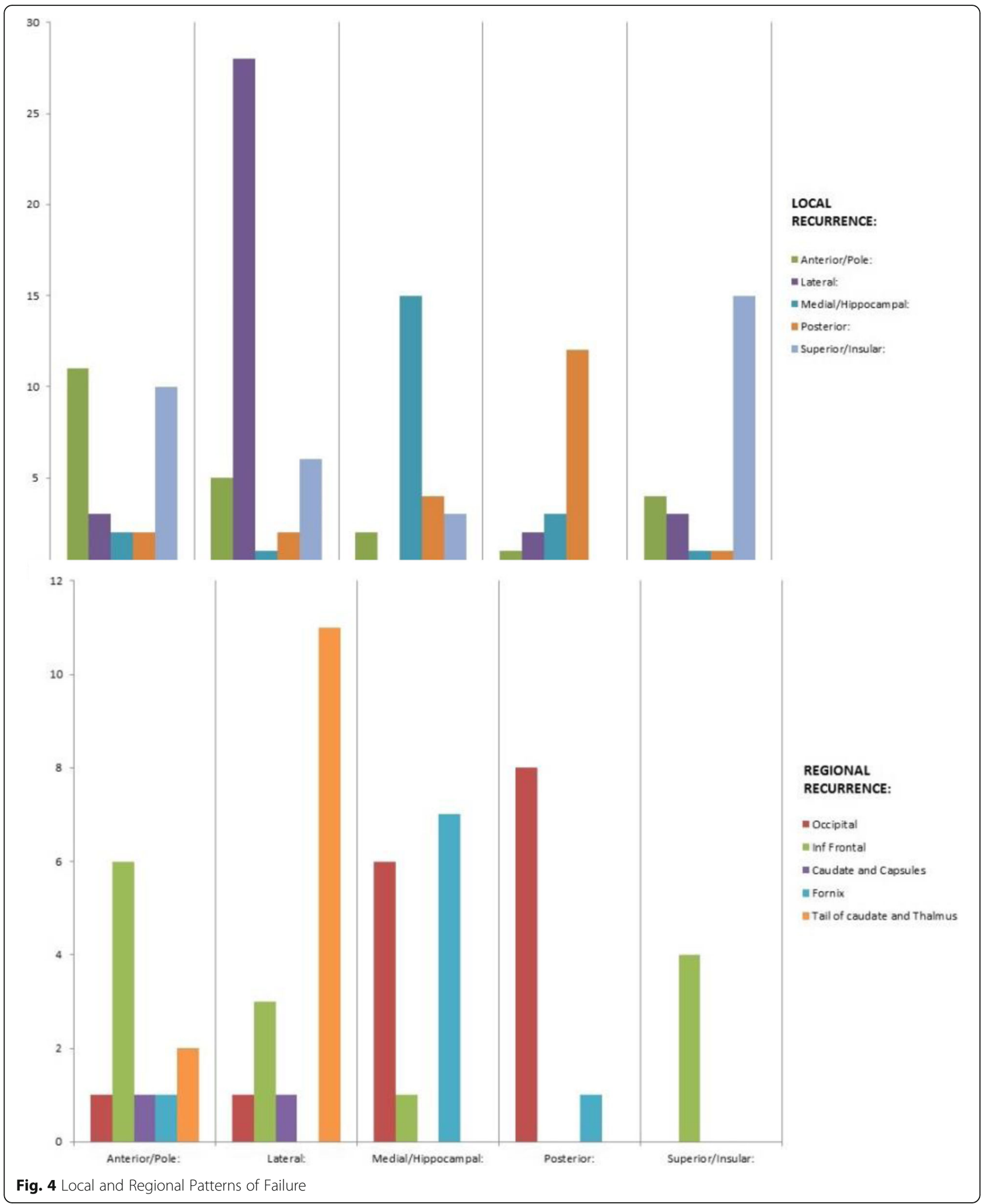

irradiated brain is believed to be associated with development of these complications [35], and treating smaller volumes should theoretically reduce these effects. As such, there is a need to improve tumour target delineation and differentiate between normal brain tissue as this may improve local control and reduce neural toxicity. 


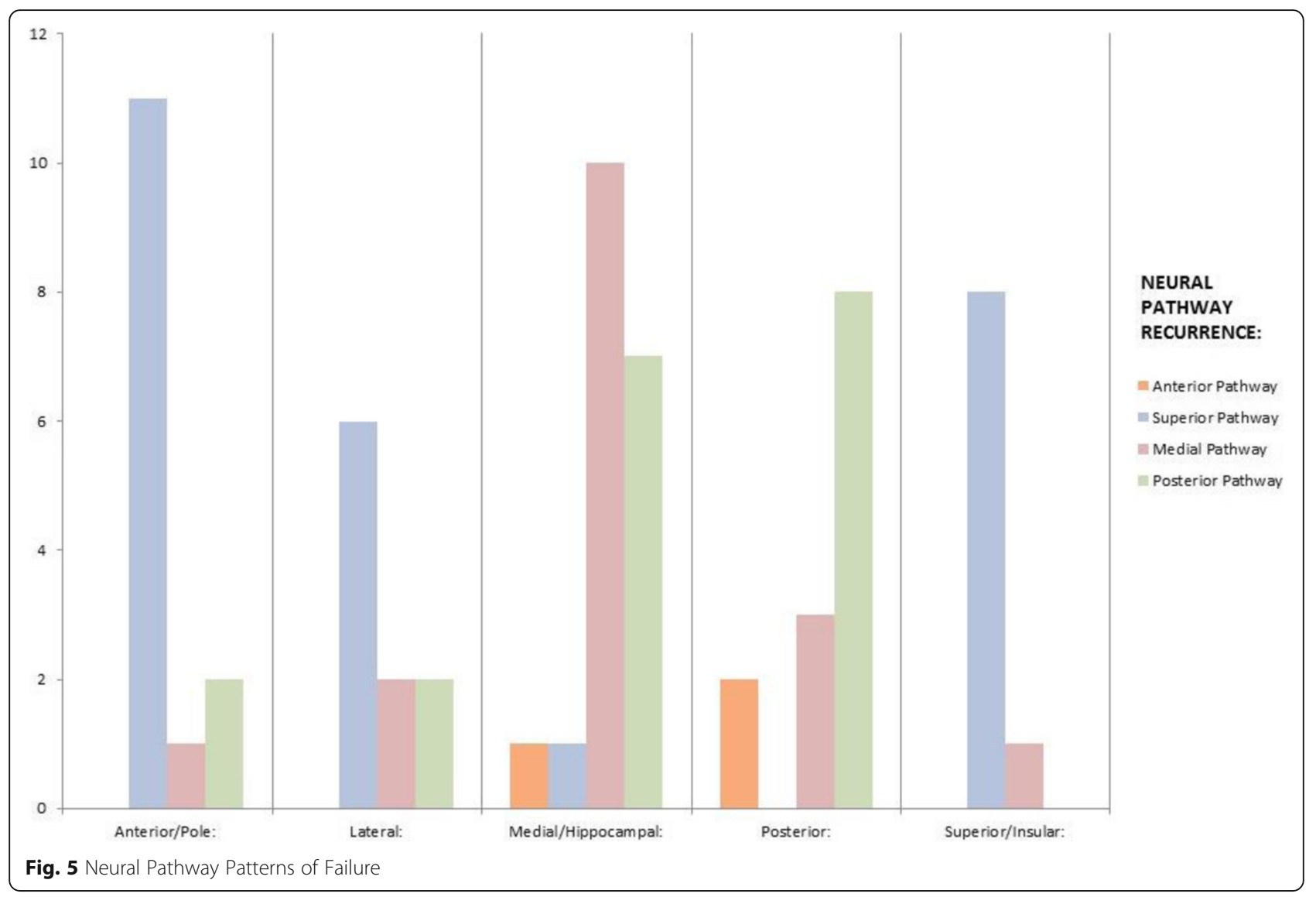

Whilst a component of local failure remains the main site of failure in the majority of patients, $15-20 \%$ of patients have an isolated regional or distant failure that appears to be related to tract involvement. Similarly patients with relatively sensitive tumours such as those with MGMT methylation, may have a proportionally greater rate of isolated tract associated regional or distant failure. This study showed 19\% of TL tumours had neural pathway involvement at time of diagnosis and $53 \%$ of patients with neural pathway failures at time of first progression. Local $(p<0.0001)$, regional $(p=0.004)$ and neural pathway failures $(p=0.005)$ were predictable and depended on the initial location of tumour within the TL. For instance patients with temporal pole tumours predominantly relapse patterns extending into the insular and inferior frontal lobe, which is connected via the uncinate fasciculus (Fig. 6). Medial tumours extending into the hippocampus had involvement tracking into the roof of the third ventricle via the fornix; or if more posterior via the cingulum to the region of the splenium (Fig. 7).

Future strategies may involve the development of new radiation target volume delineation protocols incorporating potential microscopic disease along these predictable adjacent regions may impact on disease natural history in selected patients. Given modern modulated radiotherapy techniques, this could allow for selected dose escalation along sites of greatest risk of recurrence. In other patients who will have predominantly local in-field failure, the knowledge of relapse sites and non-isotropic margin expansion that concentrates on neural pathways may limit the amount of normal tissue treated. Ideally molecular or radiological research that may identify patients at greatest risk for relapse along neural pathways would assist further in the development of such protocols.

Others have evaluated neural pathway involvement by high grade gliomas at diagnosis and recurrence using advanced MRI techniques [23, 36]. Price et al., showed

Table 3 Patterns of Failure in relation to Extent of Resection

\begin{tabular}{lllll}
\hline & Local Failure & Regional Failure & Distant Failure & Isolated Regional or Distant Failure \\
\hline Subtotal Resection or Biopsy $(n=41)$ & $80 \%$ & $63 \%$ & $43 \%$ & $20 \%$ \\
Total or Near-total Resection $(n=45)$ & $86 \%$ & $33 \%$ & $18 \%$ & $14 \%$ \\
\hline
\end{tabular}




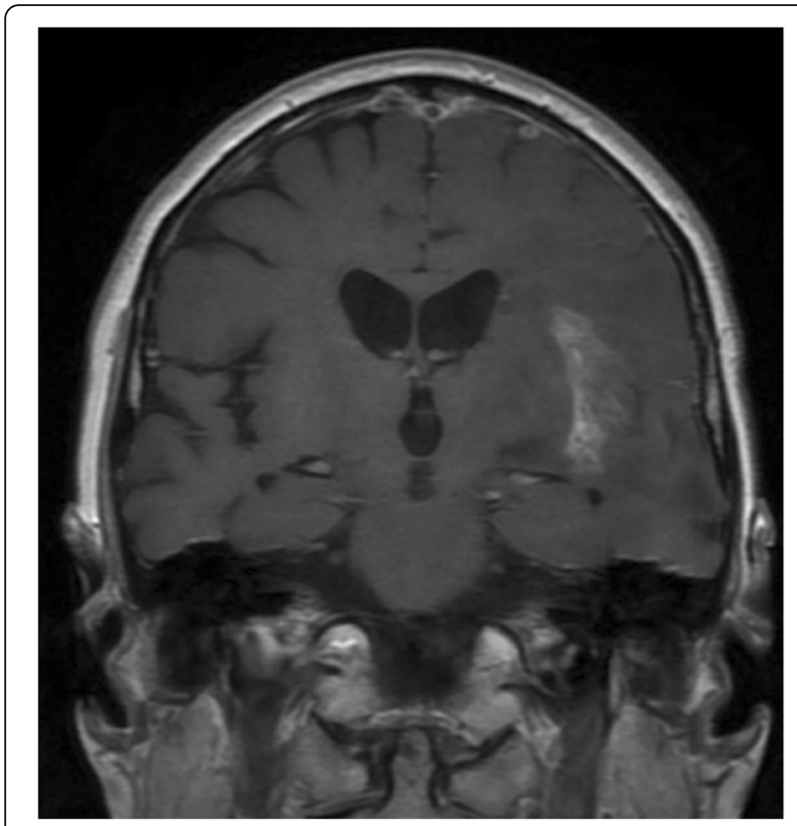

Fig. 6 Temporal pole tumour recurrence: insular lobe involvement through the uncinated fasciculus

that DTI based MRI can improve tumour delineation and assess early infiltration along neural pathways with biopsies along involved neural tracts proving positive for tumour cells [23]. DTI has also been used to assist with surgical planning of tumour resections and more recently in voluming of radiotherapy portals [37, 38]. Berberat et al. used DTI based MRI planning in treating 13 patients with GBM, showing a total reduction in treated PTVs when compared to PTVs generated by conventional T2 weighted MRI [38]. Others have shown that tract involvement can also impact survival, especially major tract involvement such as the corticospinal and spinothalamic tracts [24].

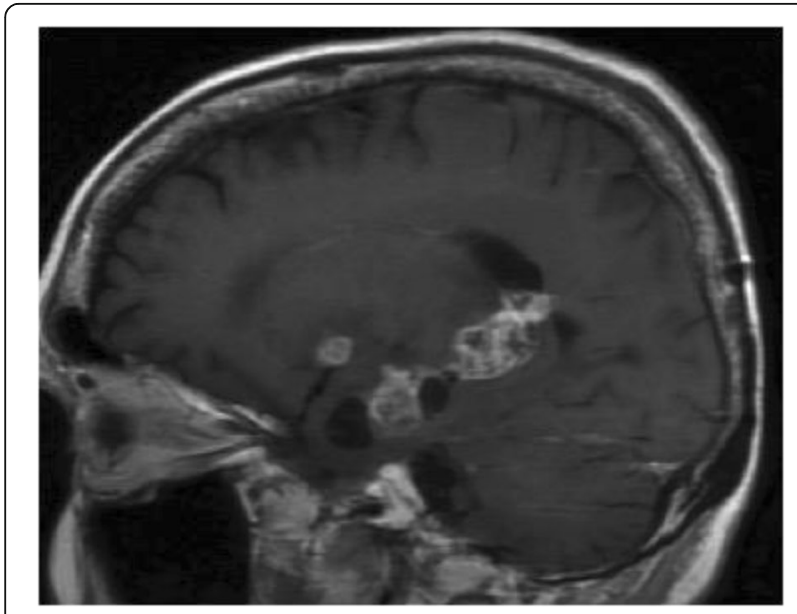

Fig. 7 Posterior medial temporal lobe tumour recurrence: tumour infiltration of the splenium through the cingulum pathway
The impact of relapse involving the neural pathways remains uncertain. In this patient cohort, salvage therapies were individualised not only based site of relapse and resectability, but also performance status at relapse, time from initial diagnosis and ability to tolerate or fund salvage systemic therapies such as Bevacizumab. The median survival from relapse to death was 6 months and this was not influenced by the pattern of relapse. Whether better coverage of neural pathways in patients whose tumours are sub-totally resected or involving neural pathways initially may delay relapse, or convert the pattern of relapse to more localised disease pattern, is uncertain. If knowledge of predictable sites of relapse is incorporated into protocols then at least significant normal tissue sites may be avoided in radiation portals, thus minimising the potential neural toxicity that may occur when combining radiation therapy with sensitising targeted therapies.

Limitations of this study include its retrospective nature with inherent problems of selection bias. Neuro-radiologists within the MDT judged tumour failure based on anatomical location and application of established neural pathway atlas information in approximating failure patterns, which may have also led to bias. Limited information was available on molecular features of the tumours analysed in this study. Only 16 patients had available results of MGMT promoter methylation status of which only 2 were positive. Another weakness involves the difficulty of distinguishing tumor progression from pseudoprogression, though we attempted to minimise the impact of this by considering subsequent changes in MRI scans and correlating this with clinical criteria for progression as per the RANO Working Group [17]. Furthermore, MRI was conducted at frequent intervals at our institution and usually at the same radiology practice which aided careful comparison and correlation with clinical findings. Additionally, our rates of locoregional and distant failure were similar to other published series. Further evaluation of neural pathway involvement could be done so in a prospective setting with functional imaging, such as DTI, with close correlation of local control and survival.

\section{Conclusions}

Despite aggressive local treatment with maximal surgical resection and adjuvant radiotherapy, most GBMs fail locally or within the high dose radiotherapy portal. Despite much evaluation, there is no consensus on tumour delineation of GBM in radiotherapy. Neural pathway involvement by GBM has been noted by others, and this study supports the theory of neural pathway infiltration as a pattern of failure. This may be an important consideration for radiotherapy delineation of tumour volumes and avoidance of normal brain tissue and may be of value for prospective evaluation. 


\section{Abbreviations}

ADC: Apparent Diffusion Coefficient; CTV: Clinical Tumour Volume; DTI: Diffusion Tensor Imaging; DWI: Diffusion Weighted Imaging; ECOG: Eastern Cooperative Oncology Group; EORTC-NCIC: ; FLAIR: Fluid Attenuated Inversion Recovery; GBM: Glioblastoma Multiforme; GTR: Gross Tumour Resection; GTV: Gross Tumour Volume; HGG: High Grade Glioma; IMRT: Intensity Modulated Radiotherapy; MDT: Multidisciplinary Tumour Board Meeting; MGMT: 06-Methylguanine DNA Methyltransferase; MR: MRI: Magentic Resonance Imaging; OS: Overall Survival; PFS: Progression Free Survival; PoF: Patterns of Failure; PTV: Planned Tumour Volume; RANO: Response Assessment in Neuro-oncology; RPA: ; RT: Radiotherapy; RTOG: ; STR: Sub-Total Resection; TL: Temporal Lobe

\section{Acknowledgements}

We thank Mary-Anne Brancato for assisting in the preparation of this report.

\section{Availability of data and materials}

The datasets generated and/or analysed during the current study are not publicly available due to confidentiality but are available from the corresponding author on reasonable request.

\section{Authors' contributions}

DJ Manuscript edits, data gathering, imaging assessment. HW Manuscript edits. DB Manuscript edits, imaging assessment. AN Manuscript edits, imaging assessment. MK Manuscript edits. LG Manuscript edits, statistical assessment. MB Manuscript edits, data gathering, imaging assessment. All authors read and approved the final manuscript.

\section{Ethics approval and consent to participate}

All procedures performed in studies involving human participants were in accordance with the ethical standards of the institutional and/or national research committee and with the 1964 Helsinki declaration and its later amendments or comparable ethical standards.

Informed consent was obtained from all individual participants included in the study.

\section{Consent for publication}

Not applicable.

\section{Competing interests}

The authors declare that they have no competing interests.

\section{Publisher's Note}

Springer Nature remains neutral with regard to jurisdictional claims in published maps and institutional affiliations.

\section{Author details}

${ }^{1}$ Northern Sydney Cancer Centre, Royal North Shore Hospital, Sydney, NSW, Australia. ${ }^{2}$ Northern Clinical School, Sydney Medical School, University of Sydney, Sydney, NSW, Australia. ${ }^{3}$ Department of Radiology, Royal North Shore Hospital, Sydney, NSW, Australia. ${ }^{4}$ Central Coast Cancer Centre, Gosford Hospital, Gosford, NSW, Australia. ${ }^{5}$ Sydney Neurooncology Group, Sydney, NSW, Australia.

\section{Received: 8 March 2018 Accepted: 12 July 2018}

Published online: 20 July 2018

\section{References}

1. Stupp R, Mason WP, van den Bent MJ, Weller M, Fisher B, Taphoorn MJB, et al. Radiotherapy plus concomitant and adjuvant Temozolomide for glioblastoma. N Engl J Med. 2005;352(10):987-96.

2. HM SR, Mason WP, van den Bent MJ, Taphoorn MJ, Janzer RC, Ludwin SK, Allgeier A, Fisher B, Belanger K, Hau P, Brandes AA, Gijtenbeek J, Marosi C, Vecht CJ, Mokhtari K, Wesseling P, Villa S, Eisenhauer E, Gorlia T, Weller M, Lacombe D, Cairncross JG, Mirimanoff RO. Effects of radiotherapy with concomitant and adjuvant temozolomide versus radiotherapy alone on survival in glioblastoma in a randomised phase III study: 5-year analysis of the EORTC-NCIC trial. Lancet Oncol. 2009;10(5):459-66.

3. Chinot OL, de La Motte RT, Moore N, Zeaiter A, Das A, Phillips H, et al. AVAglio: phase 3 trial of bevacizumab plus temozolomide and radiotherapy in newly diagnosed glioblastoma multiforme. Adv Ther. 2011;28(4):334-40.
4. Gilbert MR, Dignam JJ, Armstrong TS, Wefel JS, Blumenthal DT, Vogelbaum MA, et al. A randomized trial of bevacizumab for newly diagnosed glioblastoma. N Engl J Med. 2014;370(8):699-708.

5. Weller M, Yung WK. Angiogenesis inhibition for glioblastoma at the edge: beyond AVAGlio and RTOG 0825. Neuro-Oncology. 2013;15(8):971.

6. Stupp R, Hegi ME, Gorlia T, Erridge SC, Perry J, Hong YK, et al. Cilengitide combined with standard treatment for patients with newly diagnosed glioblastoma with methylated MGMT promoter (CENTRIC EORTC 2607122072 study): a multicentre, randomised, open-label, phase 3 trial. Lancet Oncol. 2014;15(10):1100-8.

7. Laperriere N, Zuraw L, Cairncross G. Cancer Care Ontario practice guidelines initiative neuro-oncology disease site G. Radiotherapy for newly diagnosed malignant glioma in adults: a systematic review. Radiother Oncol : J Eur Soc Ther Radiol Oncol. 2002;64(3):259-73.

8. Chang EL, Akyurek S, Avalos T, Rebueno N, Spicer C, Garcia J, et al. Evaluation of peritumoral edema in the delineation of radiotherapy clinical target volumes for glioblastoma. Int J Radiat Oncol Biol Phys. 2007;68(1):144-50.

9. Chan JL, Lee SW, Fraass BA, Normolle DP, Greenberg HS, Junck LR, et al. Survival and failure patterns of high-grade gliomas after three-dimensional conformal radiotherapy. J Clin Oncol : J Am Soc Clin Oncol. 2002;20(6):1635-42.

10. Aydin H, Sillenberg I, von Lieven H. Patterns of failure following CT-based 3D irradiation for malignant glioma. Strahlenther Onkol : Organ der Deutschen Rontgengesellschaft [et al]. 2001;177(8):424-31.

11. Oppitz U, Maessen D, Zunterer H, Richter S, Flentje M. 3D-recurrencepatterns of glioblastomas after CT-planned postoperative irradiation. Radiother Oncol : J Eur Soc Ther Radiol Oncol. 1999:53(1):53-7.

12. Coughlin C, Scott C, Langer C, Coia L, Curran W, Rubin P. Phase II, Two-arm RTOG trial (94-11) of bischloroethyl-nitrosourea plus accelerated hyperfractionated radiotherapy (64.0 or $70.4 \mathrm{~Gy}$ ) based on tumor volume (> 20 or $<$ or $=20 \mathrm{~cm}$ (2), respectively) in the treatment of newly-diagnosed radiosurgery-ineligible glioblastoma multiforme patients. Int J Radiat Oncol Biol Phys. 2000;48(5):1351-8.

13. Werner-Wasik M, Scott CB, Nelson DF, Gaspar LE, Murray KJ, Fischbach JA, et al. Final report of a phase $1 / /$ trial of hyperfractionated and accelerated hyperfractionated radiation therapy with carmustine for adults with supratentorial malignant gliomas. Radiation therapy oncology group study 83-02. Cancer. 1996;77(8):1535-43.

14. Fitzek MM, Thornton AF, Rabinov JD, Lev MH, Pardo FS, Munzenrider JE, et al. Accelerated fractionated proton/photon irradiation to 90 cobalt gray equivalent for glioblastoma multiforme: results of a phase II prospective trial. J Neurosurg. 1999;91(2):251-60.

15. Provenzale JM, McGraw P, Mhatre P, Guo AC, Delong D. Peritumoral brain regions in gliomas and meningiomas: investigation with isotropic diffusion-weighted MR imaging and diffusion-tensor MR imaging. Radiol. 2004;232(2):451-60.

16. Wen PY, Macdonald DR, Reardon DA, Cloughesy TF, Sorensen AG, Galanis E, et al. Updated response assessment criteria for high-grade gliomas: response assessment in neuro-oncology working group. J Clin Oncol : Journal Am Soc Clin Oncol. 2010;28(11):1963-72.

17. Ellingson BM, Wen PY, Cloughesy TF. Modified criteria for radiographic response assessment in glioblastoma clinical trials. Neurother : J Am Soc Exp NeuroTher. 2017;14(2):307-20.

18. Dunet V, Rossier C, Buck A, Stupp R, Prior JO. Performance of 18F-fluoroethyl-tyrosine (18F-FET) PET for the differential diagnosis of primary brain tumor: a systematic review and Metaanalysis. J Nucl Med : official publication, Soc Nucl Med. 2012;53(2):207-14.

19. Rapp M, Heinzel A, Galldiks N, Stoffels G, Felsberg J, Ewelt C, et al. Diagnostic performance of 18F-FET PET in newly diagnosed cerebral lesions suggestive of glioma. J Nucl Med : official publication, Society of Nuclear Medicine. 2013;54(2):229-35.

20. Jansen NL, Suchorska B, Wenter V, Schmid-Tannwald C, Todica A, Eigenbrod $\mathrm{S}$, et al. Prognostic significance of dynamic 18F-FET PET in newly diagnosed astrocytic high-grade glioma. J Nucl Med : official publication, Society of Nuclear Medicine. 2015;56(1):9-15.

21. Hutterer M, Nowosielski M, Putzer D, Jansen NL, Seiz M, Schocke M, et al. [18F]fluoro-ethyl-L-tyrosine PET: a valuable diagnostic tool in neuro-oncology, but not all that glitters is glioma. Neuro-Oncology. 2013;15(3):341-51.

22. Price SJ, Burnet NG, Donovan T, Green HA, Pena A, Antoun NM, et al. Diffusion tensor imaging of brain tumours at $3 \mathrm{~T}$ : a potential tool for assessing white matter tract invasion? Clin Radiol. 2003;58(6):455-62.

23. Price SJ, Jena R, Burnet NG, Hutchinson PJ, Dean AF, Pena A, et al. Improved delineation of glioma margins and regions of infiltration with the use of 
diffusion tensor imaging: an image-guided biopsy study. AJNR Am J Neuroradiol. 2006;27(9):1969-74.

24. Mickevicius NJ, Carle AB, Bluemel T, Santarriaga S, Schloemer F, Shumate D, et al. Location of brain tumor intersecting white matter tracts predicts patient prognosis. J Neuro-Oncol. 2015;125(2):393-400.

25. Colman H, Berkey BA, Maor MH, et al. Phase II Radiation Therapy Oncology Group trial of conventional radiation therapy followed by treatment with recombinant interferon-beta for supratentorial glioblastoma: results of RTOG 9710. Int J Radiat Oncol Biol Phys. 2006;66(3):818-24.

26. EORTC protocol 26082-22081. Available from: www.eortc.be/protoc/listprot. asp?kind5group\&grp51.

27. Catani M, Thiebaut de Schotten M. A diffusion tensor imaging tractography atlas for virtual in vivo dissections. Cortex; a journal devoted to the study of the nervous system and behavior. 2008:44(8):1 105-32.

28. Lee SW, Fraass BA, Marsh LH, Herbort K, Gebarski SS, Martel MK, et al. Patterns of failure following high-dose 3-D conformal radiotherapy for highgrade astrocytomas: a quantitative dosimetric study. Int J Radiat Oncol Biol Phys. 1999;43(1):79-88.

29. Mirimanoff RO, Gorlia T, Mason W, Van den Bent MJ, Kortmann RD, Fisher B, et al. Radiotherapy and temozolomide for newly diagnosed glioblastoma: recursive partitioning analysis of the EORTC 26981/22981-NCIC CE3 phase III randomized trial. J Clin Oncol : J Am Soc Clin Oncol. 2006:24(16):2563-9.

30. Nakagawa K, Aoki Y, Fujimaki T, Tago M, Terahara A, Karasawa K, et al. Highdose conformal radiotherapy influenced the pattern of failure but did not improve survival in glioblastoma multiforme. Int J Radiat Oncol Biol Phys. 1998:40(5):1141-9.

31. Brandes AA, Tosoni A, Franceschi E, Sotti G, Frezza G, Amista P, et al. Recurrence pattern after temozolomide concomitant with and adjuvant to radiotherapy in newly diagnosed patients with glioblastoma: correlation with MGMT promoter methylation status. J Clin Oncol. 2009;27(8):1275-9.

32. Niyazi M, Brada M, Chalmers AJ, Combs SE, Erridge SC, Fiorentino A, et al. ESTRO-ACROP guideline "target delineation of glioblastomas". Radiother Oncol : J Eur Soc Ther Radiol Oncol. 2016;118(1):35-42.

33. Emami B, Lyman J, Brown A, Coia L, Goitein M, Munzenrider JE, et al. Tolerance of normal tissue to therapeutic irradiation. Int J Radiat Oncol Biol Phys. 1991;21(1):109-22.

34. Torres IJ, Mundt AJ, Sweeney PJ, Llanes-Macy S, Dunaway L, Castillo M, et al. A longitudinal neuropsychological study of partial brain radiation in adults with brain tumors. Neurology. 2003;60(7):1113-8.

35. Kleinberg L, Wallner K, Malkin MG. Good performance status of long-term disease-free survivors of intracranial gliomas. Int J Radiat Oncol Biol Phys. 1993;26(1):129-33.

36. Price SJ, Jena R, Burnet NG, Carpenter TA, Pickard JD, Gillard JH. Predicting patterns of glioma recurrence using diffusion tensor imaging. Eur Radiol. 2007;17(7):1675-84.

37. Wang M, Ma H, Wang X, Guo Y, Xia X, Xia H, et al. Integration of BOLD-fMRI and DTI into radiation treatment planning for high-grade gliomas located near the primary motor cortexes and corticospinal tracts. Radiat Oncol. 2015;10:64.

38. Berberat J, McNamara J, Remonda L, Bodis S, Rogers S. Diffusion tensor imaging for target volume definition in glioblastoma multiforme. Strahlentherapie und Onkologie : Organ der Deutschen Rontgengesellschaft [et al]. 2014;190(10):939-43.

\section{Ready to submit your research? Choose BMC and benefit from:}

- fast, convenient online submission

- thorough peer review by experienced researchers in your field

- rapid publication on acceptance

- support for research data, including large and complex data types

- gold Open Access which fosters wider collaboration and increased citations

- maximum visibility for your research: over $100 \mathrm{M}$ website views per year

At BMC, research is always in progress.

Learn more biomedcentral.com/submissions 